\title{
INTEGRATING PROCUREMENT, PRODUCTION PLANNING, AND INVENTORY MANAGEMENT PROCESSES THROUGH NEGOTIATION INFORMATION
}

\author{
Giuseppe Confessore ${ }^{1}$, Silvia Rismondo ${ }^{1,2}$ and Giuseppe Stecca ${ }^{1,2}$ \\ ${ }^{1}$ Istituto di Tecnologie Industriali e Automazione - Sezione di Roma \\ Consiglio Nazionale delle Ricerche \\ Area della Ricerca Roma Tor Vergata, Via Fosso del Cavaliere, 100 \\ - 00133 Roma - ITALY \\ g.confessore@itia.cnr.it,s.rismondo@itia.cnr.it,g.stecca@itia.cnr.it \\ ${ }^{2}$ Dipartimento di Ingegneria dell 'Impresa \\ Università di Roma "Tor Vergata" \\ Via del Politecnico 1-00133 Roma - ITALY
}

\begin{abstract}
Negotiation is a basic process for the enterprise activities. In this paper we study the problem ofcreating a tool that allows a manufacturing enterprise to integrate the negotiation related to the procurement process with the production planning, and stock processes. We formulate the procurement problem as a reverse combinatorial auction, and we provide a new mathematical formulation that considers procurement, production, and stock costs.

The Combinatorial Auction approach well address the negotiation scenario in which the suppliers can exploit complementary properties (among the production parts) in order to define their offers.

In this model the objective function is given by total costs due to the purchased quantity and typology of production parts, the stock levels, and the production variations with respect to the production plan.

The winner combination produces new production plan for future periods. Therefore, the production requirement plan for the next periods, the stock levels, and the total costs will be affected.

Information elaborated by the developed tool can be also used for procurement strategy.

In this paper we show the architecture and the information exchange underlying the model. The model will be then validated by using a standard solver.
\end{abstract}

\section{INTRODUCTION}

The combination of different factors such as the reduction of the products life-cycle, the increasing products customization, the changes in the manufacturing technology, 
the geographical spreading of partners and suppliers, have a great influence on the markets, by inducing new way of work of the industrial actors (M. CamarinhaMatos L., and H. Afsarmanesh 2003). These factors have induced collaboration practices that require the introduction of new organization paradigms such as Collaborative Networked Organizations (CNO), Virtual Enterprises / Virtual Organization (VE/VO), and Professional Virtual Communities (PVC). Nevertheless, the new collaborative forms of organization have generated an increasing complexity, due mainly to the idea of consider the production system, as a part of a complex, and dynamic supply chain. In way to deal with this new scenario, companies need the help of proper supply chain management tools able to consider information flow, and physical flow along all the chain in order to exploit the potential competitive advantage. In particular, supply chain oriented tools, should cover the critical area of the supply chain from the procurement phase, to the production phase, and finally to the delivery phase. On the other hand, the exploitation of these tools is legitimated just for a restricted class of enterprises. The medium, and small enterprises (SME) are often characterize by not having stable relationships with suppliers, thus they can not be able to exploit the competitive advantage by using specified tools, that are often too expensive. Dynamism in supply chains can be effectively managed using negotiation based tools (Talluri 2002).

In this paper, respect to the conventional SCOR (Supply-Chain Operation Reference-model) model, we propose a tool able to support SME in the negotiation phase at the base of the procurement process (Bolstorff, and Rosenbaum 2003).

The main goal is to provide a tool able to take economical opportunities also in dynamic scenarios, typical of the SME. In fact, for this class of enterprises, the suppliers portfolio could change over time, either because not all the suppliers can fulfill the orders, and because the demand attributes could not met the supplier opportunities in terms, for instance, of delivery date, quantity or price.

Once the tool will be defined, then it will be inserted in a negotiation platform. The purpose of this platform is to link information due to different decisional areas of the supply chain. In this way, the enterprise could generate "best" orders, while the suppliers could generate "best" proposals, increasing the performances of the whole system. In particular, we focus on the order generation considering that the enterprise can produce orders taking into account information of the production planning, and information due to the stock process.

The auction model implemented is specialized for an enterprise that assemblies parts in order to obtain end products. The model integrates the procurement, the production, and the stock processes. The main advantage of using an auction model is due to the economical opportunities that these models give.

In the experimental phase, same hypothesis are made by considering different typologies of suppliers. In fact in this work, we consider the suppliers divided into two different sets under their supplying power. In the former set, the suppliers bid exactly the quantity the buyer wants to acquire (exact suppliers), while in the latter one they bid for batches, allowing them to exploit possible scale economy (batch suppliers). Indeed, the developed tool is able to suggest the best supplier composition for procurement strategy.

The paper is organized as follows. In Section 2, we describe the negotiation process. In Section 3, we briefly introduce the reader to the auction theory by 
emphasizing the combinatorial auction model. In Section 4, we describe the new auction model, and finally Section 5 is devoted to the experimental phase.

\section{THE NEGOTIATION RULES}

In this section we use the UML (Unified Modelling Language) diagrams in order to explain the sequence of action describing a negotiation process. Follow we show some of scenarios that a negotiation thread could produce.

We consider two different typologies of actors: the buyer and the suppliers (typically more than one). The negotiation starts when the buyer triggers a call for offer (CFO) for a set of products based on its requirements. The suppliers can accept the call for offer specifications or they can change some negotiation parameters according to their information. The buyer can either accept the negotiation parameters variation, or can change them again. The negotiation process ends when the actors reach an agreement or when the buyer forces some of the parameters, in this way some of suppliers could be out of negotiation.

If the buyers accepted the suppliers counter-offers, the agreement is formalized in a contract.

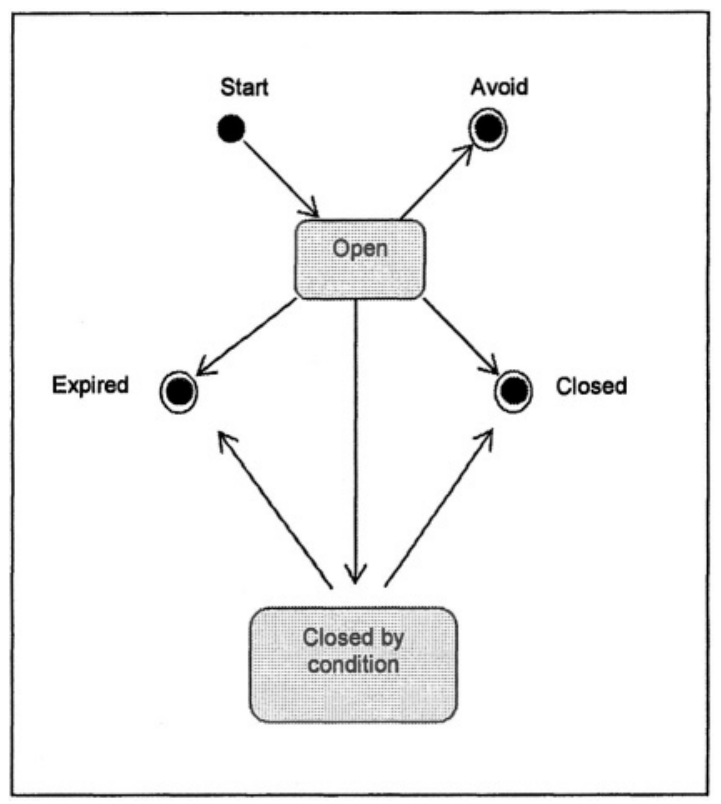

Figure 1 - State Chart Diagram of a contract.

To best understand the negotiation process, we show in Figure 1 the UML Statechart Diagram of a contract definition in a general negotiation process. When 
the buyer issue a CFO, the suppliers can make a counter-offer, opening a thread of negotiation. This thread consists of possible states described as follow.

After the buyer issues a CFO, the negotiation between the buyer, and the supplier is called open. There are a number of negotiations open (i.e., threads) such is the number of suppliers that reply to the CFO. If the buyer accepts one of the suppliers reply, then the negotiation is called closed. Otherwise, from the state open is possible to reach the state negotiation avoid. If this situation occurs no counter proposal are accepted.

The negotiation can be also in the state closed by condition. This means the buyer accepts one of the supplier counter-proposal, but the actual counter-proposal acceptance depends on another on going negotiation thread. In such situation two scenarios are possible: The negotiation ends, since the buyer concludes the negotiation at the higher level; the negotiation is in a waiting state, since it is not possible to notify the counter-proposal acceptance. In this situation the negotiation is considered as closed when the time of negotiation does not respect the time constraints. Then, the negotiation is called expired.

Therefore in this paper we refer to a classical negotiation protocol. On the other hand we focus on design a negotiation tool able to formulate CFO that consider the procurement requirements, and include also information from the production, and from the stock processes.

The main goal of this paper is to investigate which is the impact that the information flows have on the procurement process in terms of economical benefits and opportunities for the buyer. In Figure 2, we describe how the tool acts, outline the information, and material flows.

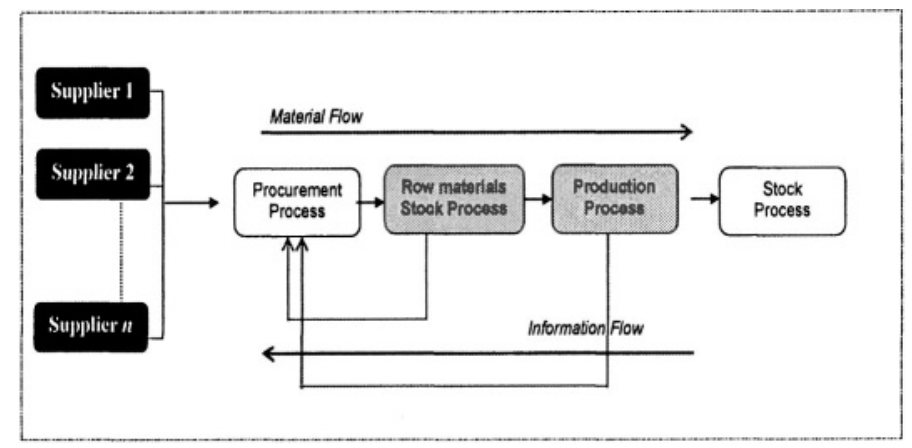

Figure 1 - The information feedback on the procurement process.

\section{THE AUCTION MODEL}

Among the known negotiation protocols, in this paper we focus on the auction that is certainly one of the main mechanisms to coordinate actors in complex systems. For the purpose of this paper, it is useful to introduce the combinatorial auction models, where the actors involved in the auction process, bid on bundles of items that exhibit complementarily and substitutability. In fact, the auction models well represent the market scenario in which it is necessary to allocate discrete, and 
complementary resource (i.e., goods) at different agents. In particular, we model the procurement process where there is a buyer who wants to obtain a set of goods at the lowest cost, and there is a set of suppliers (or sellers) who can provide these goods.

In the literature, this specific auction model is also known as reverse combinatorial auction (see Sandholm et al. 2002), and it can handle two different cases. In the first one, the buyer requires only a single unit for each good, while in the second one the buyer requires multiple units for each good. In the next, we refer to the more general multiple unit case.

Classical models, introduce a further actor, that is the auctioneer, that manages the auction process. Without loss of generality, we do not consider the auctioneer by supposing that the buyer triggers a CFO for a set of goods directly to the suppliers.

The classical reverse combinatorial auction model has the goal of minimize the buyer's cost function subject to it obtains at least the goods it requires. This can be formalise by considering a set of $n$ suppliers, and by introducing the decisional variable $\boldsymbol{x}_{\boldsymbol{j}}$ equals to 1 if supplier $j$ belongs to the winner combination, and 0 otherwise. Given $\boldsymbol{p}_{j}$ as the bid price of supplier $\boldsymbol{j}$, the objective function is defined as follows:

$$
\min \sum_{j=1}^{n} p_{j} \cdot x_{j}
$$

\subsection{The auction model extension}

In this paper, we suggest to the enterprise the exploitation of new procurement tool based on a well know coordination mechanism, that is combinatorial auction. Respect to the classical works concerning this thread of research (e.g. Sandholm (2000), Sandholm and Suri (2000)), we rather than provide new resolution algorithms, we propose a specialized model formulation able to integrate also information from the production, and from the stock processes.

In particular, the tool well manages the scenario in which there is a buyer that requires different sub-products (e.g. items) that need to be assembly in order to obtain the end products. In this setting, the buyer needs to use a tool able to pick the economical opportunities due to the items complementarity.

By introducing the decisional variable $\boldsymbol{x}_{\boldsymbol{j}}$ equals to 1 if the supplier $j$ belongs to the winner combination, and 0 otherwise. The objective function can be defined as follows:

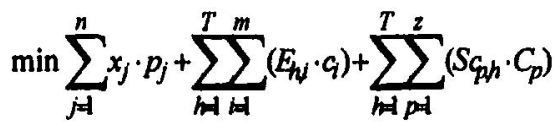

As we can see, the objective function refers to the cost minimization, given by the sum of three terms. In the formulation index $j$ refers to the suppliers, $h$ defines the time period, $i$ identifies the item or sub-product, while $p$ concerns the end product.

The first term consists in the cost due to the winner combination, as is in the classical combinatorial auction model, where $\boldsymbol{p}_{\boldsymbol{j}}$ represents supplier $j$ bid price. Second term refers to the new production allocation respect to the production 
planning. In particular, at each period $h+1$ the model considers the availability of item $i, D_{h+l, i}$ defined as follow:

$$
D_{h+1, i}=D_{h, i}+A_{h, i}+a_{h, i}-F_{h, i}
$$

$D_{h+1,1}$ is given by the availability in the previous period $h$ (i.e., $D_{h, l}$ ), the entry quantity $A_{h, l}$ of item $i$ at period $h$ (i.e., the planned quantity), the entry quantity $a_{h, l}$ of item $i$ at period $h$ dues to the combinatorial auction procurement tool, and the requirement $F_{h, l}$ of item $i$. The difference between the availability $D_{h, l}$, and the requirement $F_{h, i}$ of item $i$ at period $h$, represents the variance $E_{h, i}$. Then, in the cost function, we weigh this variance value by introducing the cost value per unit $\boldsymbol{c}_{\boldsymbol{i}}$. Latest term represents the variance value $S c_{p, h}$ dues to difference between the relocated production $Q_{h, p}$ of the end product $p$, and the production planned $\boldsymbol{P}_{h p}$. As seen before, we weight the variance $S c_{p, h}$ by introducing the cost value per unit $C_{p}$.

Referring to the second term, the model considers warehousing constraints that restrict the quantities $\boldsymbol{A}_{h, i}$, and $\boldsymbol{a}_{h, l}$ that can be received at period $h$. On the other hand, production capacity constraints force the allowable production quantities.

Following section explains the results from the experimental phase.

\section{EXPERIMENTAL RESULTS}

The main goal of this section is to identify the potentiality, and the possible application fields of the auction model analysed in this paper.

Next, we introduce the hypothesis:

- We suppose the assembly process needs 1 working day.

- We consider the demand increment at period $h$ of $10 \%$.

- The bid prices are random value uniform distributed in $[1,10]$, and they are generated by taking into account that given a possible goods composition, the price has to be higher for the exact suppliers respect to the batch ones, since we suppose the exact suppliers, by having a more flexible system for provide goods, support higher costs respect the batch suppliers.

- The suppliers composition always fulfil buyer's orders.

- The auction model is used to face positive demand picks.

In the experiment phase we consider different scenarios in which we change the $c_{i}$, and $C_{p}$ values. The goal is to identify which scenario allows the buyer to obtain economical opportunity from the demand increment by using the auction model. Moreover, whenever it is convenient to use the auction model, we identify the suppliers composition with respect to the sets identified previously.

Some preliminary considerations have to be done in relation to the cost value significance. Higher values for $c_{i}$ mean the stock costs have an huge contribution, and this, for instance, can be the situation in which goods are subject to quick obsolescence. Higher values for $\mathcal{C}_{\boldsymbol{p}}$ mean the enterprise is less flexible, and can not be convenient to meet the demand increment. 
Therefore, we analyse 4 different scenarios in which $\boldsymbol{c}_{\boldsymbol{i}}$, and $\boldsymbol{C}_{\boldsymbol{p}}$ assume Low (L), and High $(\mathrm{H})$ values. For each scenario, we generate randomly 10 different instances considering 20 items that have to be assembled, and 10 periods.

In the Table 1, we summarize the results by underlining for each scenario the percentage of times in which the buyer uses the auction, and if it exploits the auction which is the combination of suppliers respect the exact, and batch suppliers. In each cell, we introduce two pie charts. The one on the left identify the percentage of times in which the buyer uses the auction model (the portion in grey). Clearly, the white portion represents the percentage in which he/she does not use the auction model. The little pie charts at the right identify the suppliers composition.

Table 1: Scenario comparison.

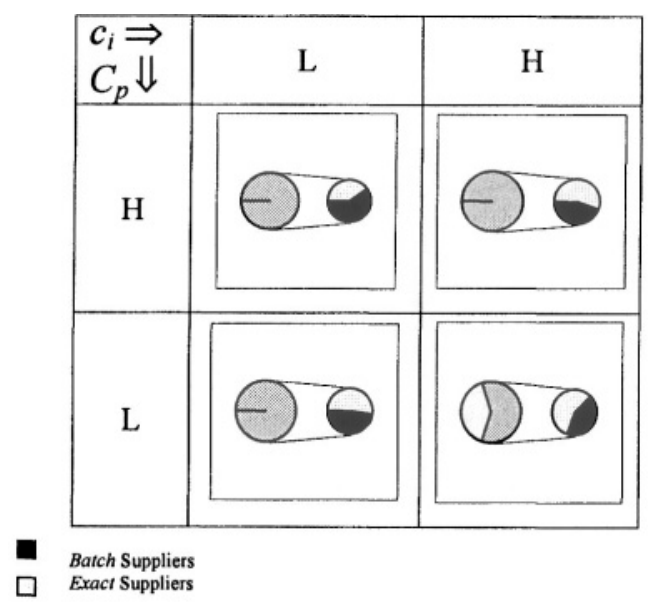

Although our investigations have clearly not been exhaustive, it is already apparent that the correct sizing of the stock, and production costs have a great influence on the performance of the tool based on the inverse combinatorial auction model. On the other hand, the tool can be very easily adapted to a specific productive environment.

\section{CONCLUSIONS}

In this paper, we provide a new combinatorial auction model able to manage the procurement process for an assembly based production system. In the model the objective function is given by the minimization of total costs due to the purchased quantity of production parts, the stock levels and the production variations with respect to the production plan. We analyse different scenario underling the strong influence of the production, and stock costs on the model performances. 
In the future, we plan to upgrade the tool by integrating it on a negotiation platform and by testing it on different industrial context.

\section{REFERENCES}

1.Bolstorff P., R. Rosenbaum. Supply Chain Excellence: A Handbook for Dramatic Improvement Using the SCOR Model. AMACOM editor 2003.

2.Camarinha-Matos L.M., and H. Afsarmanesh. A Roadmap For Strategic Research On Virtual Organizations. Proceedings of 4th IFIP Working Conference on Virtual Enterprise PRO-VE'03 2003, Processes and Foundations for Virtual Organizations, Kluwer Academic Publishers, 33-46.

3.Sandholm, T. Approaches to winner determination in combinatorial auctions. Elsevier Decision Support System 2000, 28, 165-176.

4. Sandholm, T., and S. Suri. Improved Algorithms for Optimal Winner Determination in Combinatorial Auctions and Generalizations. In: Proc. of National Conference on Artificial Intelligence 2000, 9097.

5. Sandholm, T., S. Suri, A. Gilpin, and D. Levine. Winner Determination in Combinatorial Auction Generalizations. In: Proc. of the First International Conference on Autonomous Agents and MultiAgent Systems 2002.

6. Talluri S. A buyer-seller game model for selection and negotiation of purchasing bids. European Journal of Operational Research 2002, 143. 171-180 